\title{
Overview of traumatic cardiac arrest victims' organ donation in France
}

Purpose: Organ donation is an issue which raises several ethical, economic and logistic aspects. In France, according to the law of August, $2^{\text {nd }} 2005$, a new source of organ donor was opened: Organ donations from patients deceased of cardiac arrest (ODDCA). Patients deceased following a traumatic cardiac arrest (TCA) are often targeted as potential donors. This study purpose is to characterize TCA deceased patients which are subjects of organ retrieval and to state if they really had all required criteria for theoretically been part of ODDCA program.

Results: On 3898 TCA, 48 (1.2\%) were subjects of organ retrieval. Among them, $13(27.1 \%)$ were included as nonbeating heart organ donors (donors deceased on scene) and 35 (72.9\%) were included as brain-death donors (BDD).

\begin{tabular}{|c|c|c|}
\hline & $\begin{array}{c}\begin{array}{c}\text { ODDCA } \\
\mathrm{N}=13\end{array} \\
\end{array}$ & $\begin{array}{l}\text { BDOD } \\
\mathrm{N}=35 \\
\end{array}$ \\
\hline Gender: male n(\%) & $12(92,3)$ & $28(80,0)$ \\
\hline Age: M[Q1;Q3] (years) & $33[20 ; 38]$ & $26[18 ; 38]$ \\
\hline Maastricht I n(\%) & $11(84,6)$ & NA \\
\hline Maastricht II n(\%) & $2(15,4)$ & NA \\
\hline Penetrating trauma $\mathrm{n}(\%)$ & $2(15,4)$ & $3(8,6)$ \\
\hline No-flow M[Q1;Q3] (min) & $7[0 ; 13]$ & $9[0 ; 15]$ \\
\hline T0-Hospital admission M[Q1;Q3] (min) & $66[32 ; 94]$ & $79[28 ; 111]$ \\
\hline
\end{tabular}

7 donors were younger than 18 (1 ODDCA, 6 BDD) and 5 were older than 55 (1 ODDCA, 4 BDD).
Joséphine Escutnaire, Valentine Baert, Christian Vilhelm, JeanBaptiste Marc, Evgéniya Babykina, Pierre-Yves Gueugniaud, Hervé Hubert, GR-RéAC

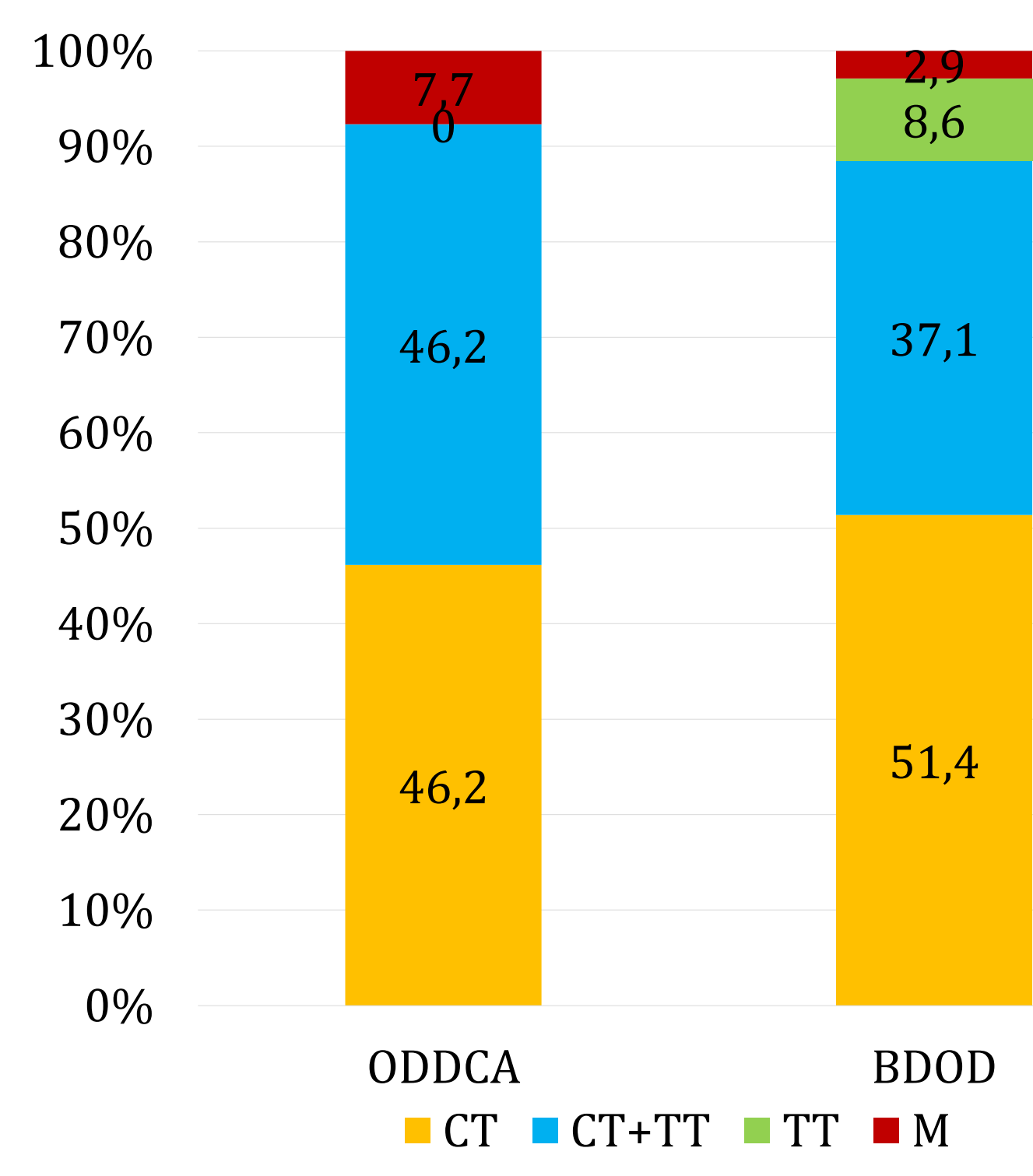

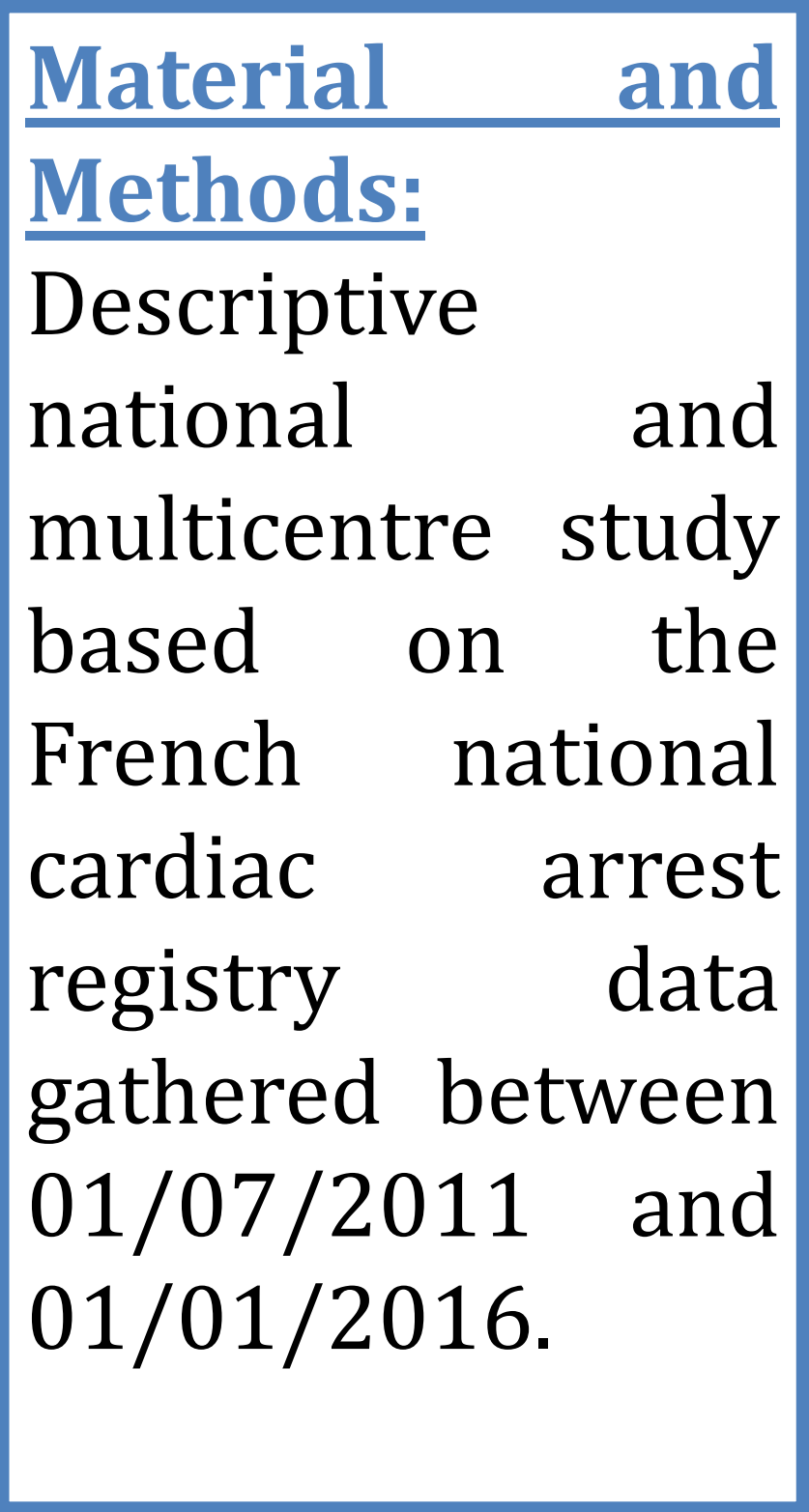

Conclusion: Organ donation in TCA is almost as frequent as Day 30 survival in France. This frequency is even higher if compatibilized on organ donation compatible TCA. Globally, included patients matched requirements expect on age criterion. Taking patients profiles into account, these results should encourage professionals to keep this issue in mind while caring refractory cardiac arrests or post-resuscitation brain death occurrence. 